\title{
Lipoprotein(a) Binds to C-terminal Lysine Residues of Recombinant Enolase Derived from Group A Streptococcus
}

\author{
Liping $\mathrm{Xu}^{1,2}$, Wencheng Bai ${ }^{1}$, Zhixing $\mathrm{Ji}^{1,3}$, Xiaoyan Dai ${ }^{1,4}$, Yun Pang ${ }^{1,5}$, Feng Huo ${ }^{6}$, Wenlong $\mathrm{Li}^{1}$, \\ Runlin Han ${ }^{1, *}$
}

${ }^{1}$ Research Center of Plasma Lipoprotein Immunology, Inner Mongolia Agricultural University, Huhhot, China

${ }^{2}$ College of Basic Medicine, Inner Mongolia Medical University, Huhhot, China

${ }^{3}$ Jining No. 1 Middle school, Jining, China

${ }^{4}$ Tianjin Institute of Scientific and Technical Information, Tianjin, China

${ }^{5}$ Inner Mongolia Yili Industrial Group Co., Ltd, Huhhot, China

${ }^{6}$ College of Life Science, Inner Mongolia Agricultural University, Huhhot, China

\section{Email address:}

han-runlin@163.com (Runlin Han)

\section{To cite this article:}

Liping Xu, Wencheng Bai, Zhixing Ji, Xiaoyan Dai, Yun Pang, Feng Huo, Wenlong Li, Runlin Han. Lipoprotein(a) Binds to C-terminal Lysine Residues of Recombinant Enolase Derived from Group A Streptococcus. American Journal of Clinical and Experimental Medicine.

Vol. 3, No. 5, 2015, pp. 327-331. doi: 10.11648/j.ajcem.20150305.33

\begin{abstract}
The biological function of lipoprotein(a) [Lp(a)] remains elusive although it was identified in 1963 . We previously hypothesized that $\mathrm{Lp}$ (a) might inhibit pathogens from hijacking host plasminogen (Plg) since apolipoprotein(a) [Apo(a)], a unique protein in $\mathrm{Lp}(\mathrm{a})$, shares a high homology with Plg. We demonstrate that $\mathrm{Lp}(\mathrm{a})$ bound to recombinant Streptococcal $\alpha$-enolase (rSEN), which is a surface Plg receptor on group A Streptococcus (GAS). However, recombinant C-terminal lysines-deleted variant of enolase (rSEN $\Delta 434-435$ ) did not bind to Lp(a). Moreover, epsilon-aminocaproic acid (EACA), a lysine analog, significantly inhibited the binding of rSEN to Lp(a). Collectively, Lp(a) via its LBS bound to the C-terminal lysines of rSEN. In addition, Lp(a) only competitively blocked the Plg-rSEN interaction but not Plg-rSEN $\Delta 434-435$ interaction since Plg could also bind to the internal lysine residue of $\alpha$-enolase. The preliminary study indicated that Lp(a) also interacted with GAS, consequently competitively inhibiting the Plg-GAS binding to some extent. Therefore, Lp(a) might play a limited role in preventing GAS infection since it only partially inhibited the pathogen from recruiting host Plg.
\end{abstract}

Keywords: Lipoprotein(a), Group A Streptococcus, Plasminogen, $\alpha$-Enolase

\section{Introduction}

It is well known that Group A Streptococcus (GAS) is a human pathogen causing a broad range of diseases including tonsillitis, pharyngitis, impetigo, mastitis, and sequelae such as rheumatic fever and glomerulonephritis [1]. GAS expresses a number of virulence factors including cell-associated and/or secreted molecules. Of these, PAM (plasminogen-binding group A Streptococcal M-like protein) [2], Streptococcal $\alpha$-enolase (SEN) and glyceraldehyde-3-phosphate dehydrogenase (GAPDH/Plr/SDH) [3,4] are thought to be major plasminogen (Plg) receptors on the GAS surface. GAS uses these receptors to secure human Plg on its surface subsequently activates bacterium-associated Plg to plasmin by its Plg activator (streptokinase) and/or host activators
(uPA and tPA) [5]. This bacterial cell-associated plasmin may facilitate the dissemination of GAS [4,6]. In addition, SEN has been demonstrated to contain several lysine residues including two C-terminal and two internal lysine residues [7], which are responsible for the binding to Plg.

$\mathrm{Lp}(\mathrm{a})$ is one unique plasma lipoprotein in humans, Old World nonhuman primates, and the European hedgehog, containing one molecule of apolipoprotein(a) [Apo(a)] covalently bound to apolipoprotein B100 (apoB100) of low density lipoprotein (LDL) via a disulfide bridge. Increased $\mathrm{Lp}(\mathrm{a})$ is a risk factor for cardiovascular disease via both atherogenic and thrombotic mechanisms [8]. However, the biological function of $\mathrm{Lp}(\mathrm{a})$ is still unknown although $\mathrm{Lp}(\mathrm{a})$ has been studied extensively since it was identified in 1963 [9-11]. Besides other domains Apo(a) contains ten classes of Plg kringle IV-like domains designated $\mathrm{KIV}_{1-10}$ [12], of which 
$\mathrm{KIV}_{10}$ contains a relatively strong LBS. $\mathrm{KIV}_{10}$ may competitively inhibit the binding between $\mathrm{Plg}$ and plasmin-modified fibrinogen [13].

We investigated whether Lp(a) can bind to SEN expressed by GAS, and if this binding can interfere the binding of SEN to Plg.

\section{Materials and Methods}

\subsection{Bacterial Cultures}

M6-type GAS (CMCC32175) was obtained from the China Medical Culture Collection Center. GAS was streaked on the nutrient broth agar containing 5\% sheep blood and was incubated for 24 hours at $37^{\circ} \mathrm{C}$. The single colony of GAS on the plate was inoculated into Todd-Hewitt broth supplemented with $0.2 \%$ Yeast extract (Oxoid, Hampshire, England) (THY medium) and was grown under 5\% $\mathrm{CO}_{2}$, $37^{\circ} \mathrm{C}$ for 17 hours. GAS cell were collected by centrifugation at $6000 \times \mathrm{g}$ for $10 \mathrm{~min}$ at $4{ }^{\circ} \mathrm{C}$, and cell pellet was washed twice with PBSA. The GAS cell suspensions in PBSA were used for the following experiments. Escherichia coli was grown at $37^{\circ} \mathrm{C}$ in Luria-Bertani (LB) broth (Tryptone 10 $\mathrm{g} \cdot \mathrm{L}^{-1}$, Yeast extract $5 \mathrm{~g} \cdot \mathrm{L}^{-1}$ ), and LB agar was used as a solid medium. Ampicillin at $100 \mathrm{ig}^{-1} \cdot \mathrm{ml}$ (Bio Basic Inc, Ontario, Canada) was used for selection markers.

\subsection{Recombinant Proteins}

Recombinant streptococcal $\alpha$-enolase (rSEN) and C-terminal lysine residues-truncated variant of $\alpha$-enolase (rSEN $\triangle 434-435)$ were produced in $E$. coli using the $6 \times$ Histag pASK-IBA37 expression vector (IBA-GmbH, Göttingen, Germany). Briefly, DNA fragment encoding $\alpha$-enolase was extracted from M6-type GAS ATCC12373 and amplified by PCR (Primers listed in Table1). After digestion with BsaI (New England Biolabs, MA, USA) the DNA fragments were cloned into the vector pASK-IBA37. E. coli BL21 strain-harboring plasmid constructs was grown in the presence of ampicillin and protein expression was induced during the exponential growth with anhydrotetracycline for 3 hours. Recombinant proteins were extracted by FastBreak Cell Lysis Reagent (Promega, WI, USA) and were purified by affinity chromatography with TALON metal affinity resins (Clontech, CA, USA). The purities of rSEN and rSEN $\triangle 434-435$ were analyzed with SDS-PAGE, and the activities of them were also detected using methods described previously [3].

Table 1. Primers used for cloning $\alpha$-enolase gene.

\begin{tabular}{ll}
\hline & Primer sequences (BsaI recognition site underlined) \\
\hline \multirow{2}{*}{ rSEN } & Forward:ATGGTAGGTCTCAGCGCATGTCAATTATT \\
& ACTGATGTATACGC \\
& Reverse:ATGGTAGGTCTCATATCATTTTTTTAAGTT \\
& ATAGAATGATTTGATAC \\
& Forward:ATGGTAGGTCTCAGCGCATGTCAATTATT \\
rSEN $\Delta 434-435$ & ACTGATGTATACGC \\
& Reverse:ATGGTAGGTCTCATATCATAAGTTATAGAA \\
& TGATTTGATACCTTTG \\
\hline
\end{tabular}

\subsection{Affinity Chromatography-Binding Assay}

Two columns were packed with $0.2 \mathrm{ml}$ of TALON metal affinity resin (50\% suspension) and equilibrated with wash buffer (Buffer W: $50 \mathrm{mM} \mathrm{NaH}_{2} \mathrm{PO}_{4}, 300 \mathrm{mM} \mathrm{NaCl}, 1 \mathrm{mM}$ PMSF, $0.01 \% \mathrm{NaN}_{3}, \mathrm{pH} 8.0$ ). To this, $50 \mu \mathrm{g} \mathrm{rSEN}$ was loaded onto one column and $50 \mu \mathrm{g}$ rSEN $\Delta 434-435$ was loaded onto another column. After washing with 8 column volume (CV) of buffer W, $5 \mu \mathrm{g}$ Lp(a) (Biomedical Technologies Inc., MA, USA) was applied onto the columns, respectively. Columns were washed with $8 \mathrm{CV}$ of buffer $\mathrm{W}$ and putative complexes of $\mathrm{rSEN}$ or $\mathrm{rSEN} \Delta 434-435$ with $\mathrm{Lp}$ (a) were eluted with elution buffer $\left(50 \mathrm{mM} \mathrm{NaH} \mathrm{PO}_{4}, 300 \mathrm{mM} \mathrm{NaCl}, 150 \mathrm{mM}\right.$ imidazole, $1 \mathrm{mM}$ PMSF, $0.01 \% \mathrm{NaN}_{3}, \mathrm{pH} 7.0$ ). The proteins in elutes were precipitated with $10 \%$ trichloroacetic acid (TCA) for $1 \mathrm{~h}$ on ice. Following centrifugation $(13000 \times \mathrm{g}$, $10 \mathrm{~min}$ ), the pellets were resuspended in $1 \mathrm{M}$ Tris- $\mathrm{HCl} \mathrm{pH}$ 8.0 buffer and analyzed by SDS-PAGE followed by immunoblotting. Electroblotting was performed at a constant voltage of $30 \mathrm{~V}$ for $1 \mathrm{~h}$ to transfer rSEN variants and at a constant current of $300 \mathrm{~mA}$ for $3 \mathrm{~h}$ to transfer Apo(a). Immunodetection of Apo(a) was carried out with a goat anti-Apo(a) antibody (Biomedical Technologies Inc., Mrkham, Canada) followed by HRP conjugated donkey anti-goat secondary antibody (R\&D Systems, MN, USA). Immunodetection of $\mathrm{rSEN}$ or $\mathrm{rSEN} \triangle 434-435$ was performed with self-made rabbit anti-rSEN antibody followed by HRP conjugated goat anti-rabbit IgG (Bios, Beijing, China). Detection was performed with chemiluminescence reagent (Tiangen, Beijing, China).

\subsection{Enzyme-Linked Immunosorbent Assay for the Protein-Protein Interactions}

The ELISA was performed at room temperature. rSEN or rSEN $\Delta 434-435(100 \mu \mathrm{l}$ of $2 \mu \mathrm{g})$ was coated onto microplate wells (Grierner bio-one, Frickenhausen, Germany) for $1.5 \mathrm{~h}$. Following washes with TBST (Tris-buffered saline and 0.05\% Tween 20) the wells were blocked with $1 \%$ BSA in TBST for 1.5h. $100 \mu \mathrm{l}$ of $0.01,0.05$ and $0.1 \mu \mathrm{g} \mathrm{Lp}$ (a) or Plg (R\&D Systems, MN, USA) or LDL in TBST was added into the wells and incubated for $1.5 \mathrm{~h}$. For inhibition experiments, the mixture Lp(a) and Plg was added. After three washes with TBST, 100 $\mu \mathrm{l}$ of diluted goat anti-Apo(a) antibody was added to the corresponding wells and incubated for $1.5 \mathrm{~h}$. HRP-conjugated donkey anti-goat was used as secondary antibody and the reaction was developed with TMB substrate (Tiangen, Beijing, China). After $15 \mathrm{~min}$ of color development, the stop solution (8.5 $\mathrm{M}$ acetic acid, $2.5 \mathrm{M} \mathrm{H}_{2} \mathrm{SO}_{4}$ ) was added and the absorbance was recorded at $450 \mathrm{~nm}$. For some experiments monoclonal mouse anti-Plg antibody and HRP-conjugated goat anti-mouse were used to detect Plg.

\section{Statistical Analysis}

All tests were performed in triplicates, and results were expressed as mean \pm SD. Statistical significance was calculated using two-tailed Student's $t$-test for paired data. 
Differences with $p<0.05$ were considered statistically significant $(* P<0.05, * * P<0.01)$.

\section{Results}

\subsection{Lp(a) Via its LBS Binds to rSEN}

A

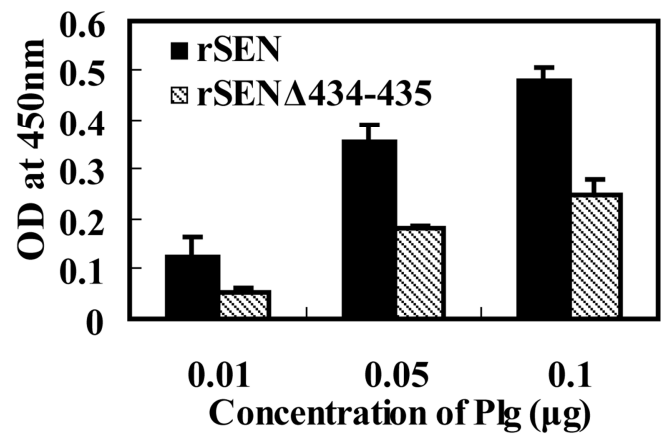

B

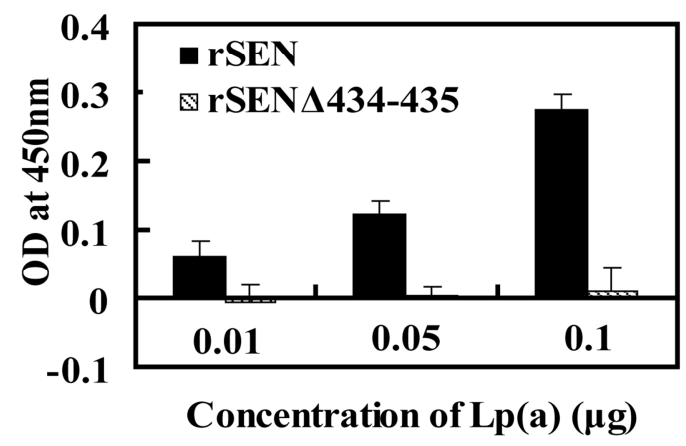

C

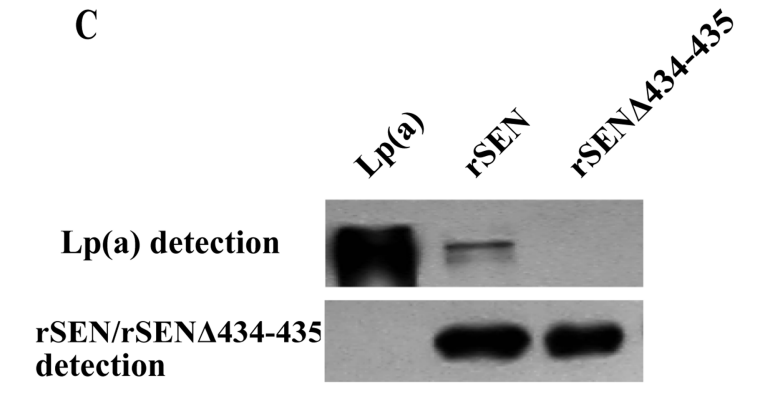

Figure 1. The binding of rSEN and rSENA434-435 to Plg and Lp(a).

ELISA was used to detect the binding of rSEN and rSEN $\triangle 434-435$ to Plg (A) and $\mathrm{Lp}$ (a) (B). $2 \mu \mathrm{g} /$ well of rSEN and rSEN $\triangle 434-435$ were immobilized into the microplate wells and different amounts of Plg or $\mathrm{Lp}(\mathrm{a})$ were added into the wells. Bound ligands were detected with corresponding antibodies. Color was developed with an HRP substrate and absorbance was recorded at 450 $\mathrm{nm}$. Mean absorbance values \pm SD from triplicate wells are shown after subtracting the OD values of the controls. For Affinity chromatography analysis (C), two columns were packed with $0.2 \mathrm{ml}$ of the TALON metal affinity resin and equilibrated with wash buffer. $50 \mu \mathrm{g}$ of rSEN 50 or rSEN $\triangle 434-435$ was loaded onto the column. After washing $5 \mu \mathrm{g}$ of $\mathrm{Lp}(\mathrm{a})$ was applied and columns were washed and putative complexes of rSEN or rSEN $\triangle 434-435$ with $\mathrm{Lp}$ (a) were eluted with elution buffer. The proteins in elutes were TCA-precipitated and analyzed by SDS-PAGE followed by immunoblotting.
Our results showed that rSEN bound to Plg (Fig. 1A), whereas rSEN $\triangle 434-435$ still had a less binding capacity to Plg. However, Lp(a) could only bind to rSEN with concentration-dependent manner but not to rSEN $\Delta 434-435$ (Fig. 1B). Moreover, the pull-down followed by Western blotting assay confirmed above Lp(a)-rSEN interaction (Fig. 1C). Therefore, Lp(a) via its LBS might interact with the C-terminal lysine residues of rSEN.

The binding inhibited by EACA (a lysine analog) was also tested to further explore the mechanism of $\mathrm{Lp}(\mathrm{a})-\mathrm{rSEN}$ interaction. The results showed that EACA significantly inhibited not only Plg-rSEN binding but also Lp(a)-rSEN interaction (Fig. 2). Taken together, LBS in $\mathrm{Lp}(\mathrm{a})$ and C-terminal lysine residues in rSEN were responsible for the protein-protein interaction.

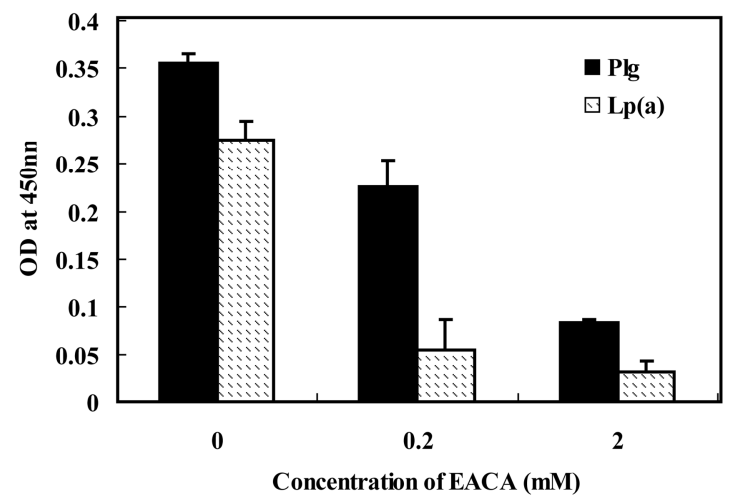

Figure 2. The effect of EACA on the interaction of rSEN with Plg and Lp(a).

After rSEN was immobilized into microplate wells, Plg or Lp(a) $(0.1 \mu \mathrm{g})$ the different concentration of EACA was added to the wells. After washing bound ligands were detected with corresponding antibodies. The color was developed with an HRP substrate and OD at 450nm was recorded. Mean absorbance values \pm SD from triplicate wells were obtained after subtracting the OD values of the control wells without rSEN.

\subsection{Lp(a) Inhibits the Binding of rSEN to Plg}

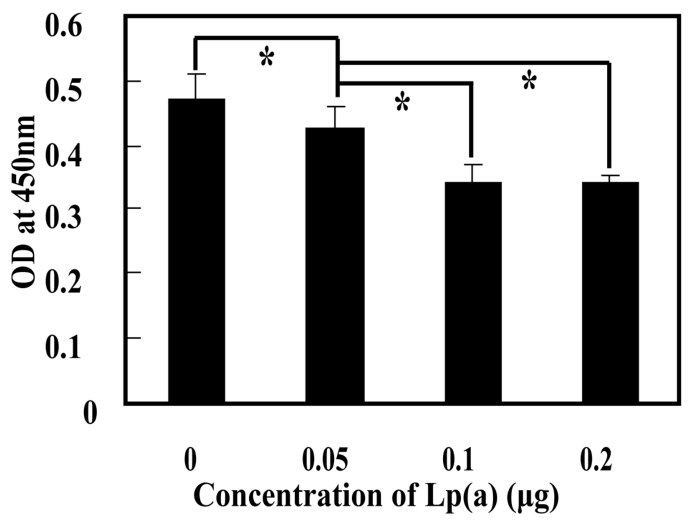

Figure 3. The effect of Lp(a) on the binding between rSEN and Plg.

After rSEN was immobilized into microplate wells, the mixtures of Plg $(0.1 \mu \mathrm{g})$ and different concentration of $\mathrm{Lp}(\mathrm{a})$ were added in the wells. After washing, bound Plg was detected with the anti-Plg antibody. The color was developed with an HRP substrate and OD at 450nm was recorded. Mean absorbance values \pm SD from triplicate wells were obtained after subtracting the OD values of the control wells without rSEN. 
Now that Plg and Lp(a) via their LBS could interact with rSEN, we also tested if $\mathrm{Lp}$ (a) competitively inhibited the binding of rSEN to Plg. The results showed that Lp(a) (Fig. 3) could significantly decrease the Plg-rSEN interaction. Lp(a) might only inhibit the binding of Plg to C-terminal lysine residue(s) but not to internal lysine residues of rSEN, since $\mathrm{Plg}$ could bind to both $\mathrm{C}$-terminal and internal lysine residues [7] whereas $\mathrm{Lp}(\mathrm{a})$ was only able to interact with C-terminal lysine residues of rSEN (Fig. 1).

\section{Discussion}

Numerous pathogens including bacteria [14-18], parasites [19-22] and fungus [23], recruit plasmin(ogen) through the lysine residue in their surface Plg receptors, and some even produce $\mathrm{Plg}$ activators to penetrate tissue barriers. Plg recruiting and activation is important for the GAS invasion [6, $24,25]$.

In our study, the binding of Plg to rSEN $\triangle 434-435$ is lower compared with that to rSEN, which has been demonstrated previously [3, 7]. However, Lp(a) only binds to rSEN, indicating that the C-terminal lysine residue of SEN is the only site for $\mathrm{Lp}(\mathrm{a})$ binding.

Besides, the results show that $\mathrm{Lp}(\mathrm{a})$ inhibits the binding of Plg to SEN. The competition mechanism may involved in the LBS structure in Lp(a) and Plg [26]. In our previous study, Streptococcal glyceraldehyde-3-phosphate dehydrogenase (GAPDH), which is thought to be another weak Plg receptor, could also bind to $\mathrm{Lp}(\mathrm{a})$ using recombinant GAPDH-Lp(a) interaction assay [27] since GAPDH contains C-terminal lysine residue (GeneBank Accession\#: NC_006086). Moreover, Apo(a) can inhibit the activation of Plg on human umbilical endothelial cell, THP-1 monocytes and macraphages [28]. We have hypothesized that Lp(a) might play an important role in preventing infections by inhibiting host plasmin(ogen) recruitment and Plg activition on the surface of bacteria, since Apo(a) shares a high homology with Plg and both of them contain lysine binding sites (LBS) [29]. Thus, further studies on the interactions of $\mathrm{Lp}$ (a) with other pathogens and anti-infective experiments with Apo(a) transgenic animal model are warranted.

\section{Conclusion}

The present study demonstrated that human plasma Lp(a) bound to C-terminal lysine residue of rSEN derived from GAS and subsequently inhibited the binding of SEN to Plg. Our results implied that $\mathrm{Lp}(\mathrm{a})$ might play a role in protecting host against GAS infection since $\mathrm{Lp}(\mathrm{a})$ could partially inhibit the Plg-GAS interaction.

\section{Acknowledgements}

Wencheng Bai and Liping Xu contributed equally to this study. This work was supported by the National Natural Science Foundation of China (Grant No. 30860019). The co-authors declare no conflict of interest.

\section{References}

[1] A. H. Nobbs, R. J. Lamont, H. F. Jenkinson. Streptococcus Adherence and Colonization. Microbiol. Mol. Biol. Rev., 2009, 73: 407-450.

[2] A. Berge, U. Sjobring. PAM, a novel plasminogen-binding protein from Streptococcus pyogenes. J. Biol. Chem., 1993, 268: $25417-25424$.

[3] V. Pancholi, V. A. Fischetti. alpha-enolase, a novel strong plasmin(ogen) binding protein on the surface of pathogenic streptococci. J. Biol. Chem., 1998, 273: 14503-14515.

[4] M. J. Walker, J. D. Mcarthur, F. Mckay, et al. Is plasminogen deployed as a Streptococcus pyogenes virulence factor? Trends Microbiol., 2005, 13: 308-313.

[5] M. L. Sanderson-Smith, D. M. P. De Oliveira, M. Ranson, et al. Bacterial Plasminogen Receptors: Mediators of a Multifaceted Relationship. J. Biomed. Biotechnol., 2012.

[6] S. Bhattacharya, V. A. Ploplis, F. J. Castellino. Bacterial Plasminogen Receptors Utilize Host Plasminogen System for Effective Invasion and Dissemination. J. Biomed. Biotechnol., 2012.

[7] A. J. Cork, S. Jergic, S. Hammerschmidt, et al. Defining the Structural Basis of Human Plasminogen Binding by Streptococcal Surface Enolase. J. Biol. Chem., 2009, 284: 17129-17137.

[8] E. Anuurad, M. B. Boffa, M. L. Koschinsky, et al. Lipoprotein(a): A unique risk factor for cardiovascular disease Clin. Lab. Med., 2006, 26: 751-+.

[9] L. Berglund, R. Ramakrishnan. Lipoprotein(a): An elusive cardiovascular risk factor. Arterioscler. Thromb. Vasc. Biol., 2004, 24: 2219-2226.

[10] M. L. Koschinsky. Novel insights into Lp(a) physiology and pathogenicity: more questions than answers? Cardiovasc. Hematol. Disord. Drug Targets, 2006, 6: 267-278.

[11] S. P. A. Mccormick. Lipoprotein(a): biology and clinical importance. Clin. Biochem. Reviews/AACB, 2004, 25: 69-80.

[12] J. W. Mclean, J. E. Tomlinson, W. J. Kuang, et al. cDNA sequence of human apolipoprotein(a) is homologous to plasminogen. Nature, 1987, 330: 132-137.

[13] M. N. Rahman, V. Petrounevitch, Z. Jia, et al. Antifibrinolytic effect of single apo(a) kringle domains: relationship to fibrinogen binding. Protein Eng., 2001, 14: 427-438.

[14] S. Agarwal, P. Kulshreshtha, D. B. Mukku, et al. alpha-Enolase binds to human plasminogen on the surface of Bacillus anthracis. BBA-Proteins Proteomics, 2008, 1784: 986-994.

[15] V. Chumchua, N. Pornputtapong, C. Thammarongtham, et al. Homology modeling of Mycoplasma pneumoniae enolase and its molecular interaction with human plasminogen. Bioinformation, 2008, 3: 18-23.

[16] M. C. Jobin, J. Brassard, S. Quessy, et al. Acquisition of host plasmin activity by the swine pathogen Streptococcus suis serotype 2. Infect. Immun., 2004, 72: 606-610. 
[17] K. Lahteenmaki, P. Kuusela, T. K. Korhonen. Bacterial plasminogen activators and receptors. FEMS Microbiol. Rev., 2001, 25: 531-552.

[18] J. Sha, T. E. Erova, R. A. Alyea, et al. Surface-Expressed Enolase Contributes to the Pathogenesis of Clinical Isolate SSU of Aeromonas hydrophila. J. Bacteriol., 2009, 191: 3095-3107.

[19] V. Mundodi, A. S. Kucknoor, J. F. Alderete. Immunogenic and plasminogen-binding surface-associated alpha-enolase of Trichomonas vaginalis. Infect. Immun., 2008, 76: 523-531.

[20] A. Ramajo-Hernandez, R. Perez-Sanchez, V. Ramajo-Martin, et al. Schistosoma bovis: Plasminogen binding in adults and the identification of plasminogen-binding proteins from the worm tegument. Exp. Parasitol., 2007, 115: 83-91.

[21] M. Rojas, I. Labrador, J. L. Concepcion, et al. Characteristics of plasminogen binding to Trypanosoma cruzi epimastigotes. Acta Trop., 2008, 107: 54-58.

[22] G. Vanegas, W. Quinones, C. Carrasco-Lopez, et al. Enolase as a plasminogen binding protein in Leishmania mexicana. Parasitol. Res., 2007, 101: 1511-1516.

[23] J. D. Crowe, I. K. Sievwright, G. C. Auld, et al. Candida albicans binds human plasminogen: identification of eight plasminogen-binding proteins. Mol. Microbiol., 2003, 47: $1637-1651$.

[24] H. M. Sun, U. Ringdahl, J. W. Homeister, et al. Plasminogen is a critical host pathogenicity factor for group A streptococcal infection. Science, 2004, 305: 1283-1286.

[25] A. Godier, B. J. Hunt. Plasminogen receptors and their role in the pathogenesis of inflammatory, autoimmune and malignant disease. J. Thromb. Haemost., 2013, 11: 26-34.

[26] R. Romagnuolo, K. Demarco, M. B. Boffa, et al. Apolipoprotein(a) inhibits the conversion of Glu-plasminogen to Lys-plasminogen on the surface of vascular endothelial and smooth muscle cells. J. Thromb. Haemost., 2013, 11: 377-377.

[27] X. Y. Dai, L. P. Xu, W. C. Bai, et al. The interaction between lipoprotein(a) anrecombinant glyceraldehyde-3-phosphate dehudrogenase derived from group a streptococcus (in Chinese). J. Inner Mongolia Agric. Univ., 2011, 32: 27-31.

[28] R. Romagnuolo, S. M. Marcovina, M. B. Boffa, et al. Inhibition of plasminogen activation by apo(a): role of carboxyl-terminal lysines and identification of inhibitory domains in apo(a). J. Lipid Res., 2014, 55: 625-634.

[29] R. L. Han. Plasma lipoproteins are important components of the immune system. Microbiol. Immunol., 2010, 54: 246-253. 